\title{
ß-Glucans and Post Prandial Satiety: The Role of Intestinal Hormones in Healthy Volunteers
}

Sara Baldassano ${ }^{1}$, Silvio Buscemi ${ }^{2}$, Anna Aiello ${ }^{3}$, Giulia Accardi ${ }^{3}$, Calogero Caruso ${ }^{3}$, Sonya Vasto $^{1,4 *}$

${ }^{1}$ Department of Biological Chemical and Pharmaceutical Sciences and Technologies (STEBICEF), University of Palermo, Viale delle Scienze Parco d'Orleans, Edificio 16, 90128, Palermo, Italy; sonya.vasto@unipa.it; sara.baldassano@unipa.it

${ }^{2}$ Biomedic Department of Internal and Specialistic Medicine (DIBIMIS), University of Palermo, Via del Vespro 129, 90127 Palermo, Italy; silvio.buscemi@unipa.it

${ }^{3}$ Department of Pathobiology and Medical Biotechnologies (DIBIMED), University of Palermo, Corso Tukory 211, 90134, Palermo, Italy; calogero.caruso@unipa.it; giuliabio@gmail.com; anna.aiello2903@gmail.com

${ }^{4}$ Institute of biomedicine and molecular immunology "Alberto Monroy" CNR, Via Ugo la Malfa, 153, 90146 Palermo, Italy; sonya.vasto@unipa.it

* Correspondence: sonya.vasto@unipa.it; Tel.: +39-09123897508 


\begin{abstract}
Recent interest in intestinal hormones has risen with the idea that they modulate glucose tolerance and food intake through a variety of mechanisms, and such hormones like peptide YY (PYY), ghrelin, glucagon-like peptide (GLP)-1 and 2, and cholecystokinin (CKK) are therefore excellent therapeutic candidates for the treatment of diabetes and obesity. Furthermore, in the recent years, multiple studies suggest that the microbiota is critically important for normal host functions, while impaired host microbiota interactions contribute to the pathogenesis of numerous common metabolic disorders. In this study, we considered the nutraceutical effects of $\beta$-glucans added to pasta at the concentration of $6 \mathrm{~g} \backslash 100 \mathrm{~g}$. Ten participants have been recruited and hematochemical analyses and intestinal hormones tests have been performed before and after 30 days of pasta intake. Stool specimens have been studied for Lactobacillus Fermentum, Lactobacillus acidophilus, Lactobacillus salivarius, Bifidobacterium longum, and Enterococcus faecium presence before and after 30 days of nutritional intervention. After 30 days of regular intake of pasta enriched by $\beta$-glucans results have been evaluated. In conclusion, pasta prepared from barley flour enriched with $\beta$-glucans at $6 \%$ exhibit promising responses on glucose metabolism, on intestinal hormones responses and on microbiota modification.
\end{abstract}

Key words: $\beta$-glucans; entero-endocrine system; intestinal hormones; microbiota; nutritional intervention 


\section{Introduction}

The $\beta$-glucans are soluble fibres commonly found in the cell wall of yeasts, fungi, bacteria, algae and cereals. All the $\beta$-glucans are polysaccharides consisting of linear molecules of D-glucose joined together by glycosidic bonds linear $\beta$ (1-3) and $\beta$ (1-4) and differ between them for the length and branched structures. The branches derived from the nuclear chain glycoside are highly variable and the two main groups are branching chains glycosidic $\beta(1-4)$ and $\beta$ (1-6). These ramifications appear to be specific. For example, the $\beta$-glucans of mushrooms have side branches $1 \rightarrow 6$ while those of bacteria have side branches $1 \rightarrow 4[1,2]$. The presence of the bond $\beta(1-3)$ leads to the formation of folds in the linear chain that allow water to enter; for this reason the $\beta$-glucans are classified as soluble fibres. Characteristics of $\beta$-glucans are their effect on cholesterol that depends on the ability to form a viscous layer on the surface of the small intestine that reduces the absorption of cholesterol and the bile acids. The inhibition of the reabsorption of bile acids can increase their synthesis from endogenous cholesterol thus reducing the blood levels of low density lipoprotein (LDL)-cholesterol [3]. This action has already been investigated using breakfast drinks [4], biscuits and crackers added with less of $3 \%$ of $\beta$-glucans [3]. A minimum dose of $3 \mathrm{~g}$ /day has been demonstrated to reduce the blood levels of cholesterol and consequently it decreases the risk of cardiovascular diseases [4]. In fact, the $\beta$-glucans viscous layer may have beneficial effects on glycaemia and sensations of hunger and satiety $[4,5]$. In a recent report, some of us considered the nutraceutical effects of $\beta$-glucans added to pasta $(6 \%)$. After 30 days of pasta intake, we obtained encouraging results with a significant decrease of LDL-cholesterol, interleukin (IL)-6 and advanced glycation end-product levels [6]. Therefore, the nutraceutical use of $\beta$-glucans seems to be an interesting perspective [7].

On the other hand, the effects of $\beta$-glucans on satiety have been reported in many studies, but no consensus has been reached. In a recent report, it was examined the effects of breakfasts varying in the dose of oat bran (4g or $8 \mathrm{~g} \beta$-glucans). The sensations associated with hunger and satiety were evaluated using visual analogue scales before and after ingesting the test breakfasts and every $30 \mathrm{~min}$ until $210 \mathrm{~min}$. Oat bran addition in breakfasts increased postprandial satiety [8]. However, the effects of regular intake in human of higher amounts of $\beta$-glucans on intestinal-hormones, that concur to regulate appetite/satiety, have not been defined.

Moreover, many studies demonstrated the role of gut microbiota in many inflammatory diseases, including type 2 diabetes mellitus and obesity. Two groups of beneficial bacteria are dominant in the human gut, the Bacteroidetes and Firmicutes. The relative proportion of Bacteroidetes is decreased in obese people by comparison with lean people, and this proportion increases with weight loss, indicating that obesity has a microbial component [9]. Thus, the alteration of microbiota homeostasis can be involved in the pathogenesis of diseases but it is not clear if this 
process is a consequence or a cause of it. In animal model modification of intestinal microbiota seems to affect insulin resistance and post prandial glucose response perhaps due to reduced gut permeability and therefore influencing intestinal hormones concentration. More interestingly, mice model treated with prebiotics showed increased levels some intestinal-hormones [10].

Microbiota has been already identified as a metabolic organ that could ferment non digestible dietary components. In fact, microbiota generates short chain fatty acids (SCFAs), which could affect host energy metabolism and could develop obesity by changing the hormonal secretion in the intestine. So, microbiota cyclical fluctuation in composition may trigger intestinal hormones release. In addition, in obese rats and high-fat diet-fed mice an intervention with prebiotics stimulates Bifidobacteria and reduces weight, by increasing intestinal-hormone PYY and reducing ghrelin [11]. In obese people during weight gain it is observed a decreased level of GLP-1, which is normally regulated by gut microbiota. GLP-1 influences the expression of the proglucagon (its precursor), which causes insulin resistance independent of fatty acids circulating level [12].

Thus, in the present pilot study we investigated the modification of serum concentrations of orexigenic peptide ghrelin, anorexigenic active form of GLP-1, GLP-2, CKK, and PYY. Those hormones were evaluated in the postprandial period after a challenge with a test meal constituted by pasta supplemented with $6 \% \beta$-glucans.

This study was performed in overweight and healthy individuals, before and after 30 days of pasta intake. In addition, we quantified the variation of some bacterial strains in faeces samples at the baseline and after 30 days of nutritional intervention.

\section{Results}

\subsection{Hematochemical Tests}

Physical characteristics and biochemical measurements are presented in Table 1. In particular, biochemical values did not change following the 30 days intervention with $\beta$-glucans with the exception of glucose concentrations and total cholesterol, as reported in Table 1. Body weight and body mass index (BMI) were not significantly changed at the end of the study. As observed in the previous study [6], the treatment with $\beta$-glucans induced a reduction of serum LDL-cholesterol concentrations.

Instead, in this study there were no significant alteration of high density lipoprotein (HDL)cholesterol and triglycerides concentrations or the other considered parameters, this it may be due to the small size sample. 
On the other hand, in the present study we have observed a significant reduction of glycaemic levels after the treatment, datum not observed in the previous study, likely because previously only healthy normal weight subjects were studied.

Table 1. Characteristics of the cohort and blood measurements before and 30 days after regular consumption of pasta added with $\beta$-glucans .

\begin{tabular}{lccc}
\hline & Before & After & $\mathbf{P}^{\mathbf{1}}$ \\
\hline \hline Gender (Females/Males) & $10 / 10$ & $10 / 10$ & \\
Age (years) & $26.5 \pm 2.5$ & & \\
Body weight (kg) & $84.8 \pm 8.1$ & $84.2 \pm 6.5$ & \\
BMI (kg/m2) & $27.4 \pm 3.4$ & $27.3 \pm 3.3$ & 0.05 \\
Fasting blood measurements: & & & 0.03 \\
Glucose (mg/dl) & $99 \pm 2$ & $89 \pm 9$ & 0.8 \\
Total cholesterol (mg/dl) & $175 \pm 24,7$ & $160 \pm 13,3$ & 0.03 \\
HDL-cholesterol (mg/dl) & $52 \pm 7,5$ & $52 \pm 5,1$ & 0.3 \\
LDL-cholesterol (mg/dl) & $103 \pm 20,7$ & $91 \pm 10,4$ & $82 \pm 26,3$ \\
Triglycerides (mg/dl) & $96 \pm 32,7$ &
\end{tabular}

All data are presented as means \pm S.D.

\subsection{Gut Hormone Responses}

GLP-1 (Figure 1). The consumption of test meal induced an increase in plasma GLP-1 concentrations, starting from $15 \mathrm{~min}$ up to $90 \mathrm{~min}$, peaking at $30 \mathrm{~min}$. The consumption of the $\beta$ glucans enriched pasta for 30 days significantly increased the levels of GLP-1 at 0, 30 and 60 minutes after intake. As shown by area under the curve (AUC), on the whole after 30 days treatment we observed an increased release of GLP-1. 
A

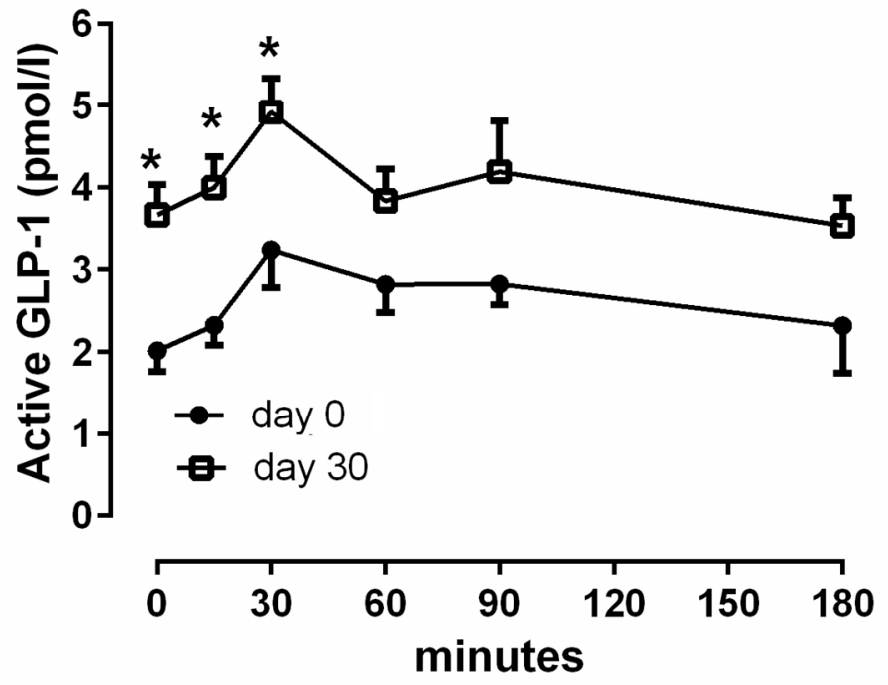

B

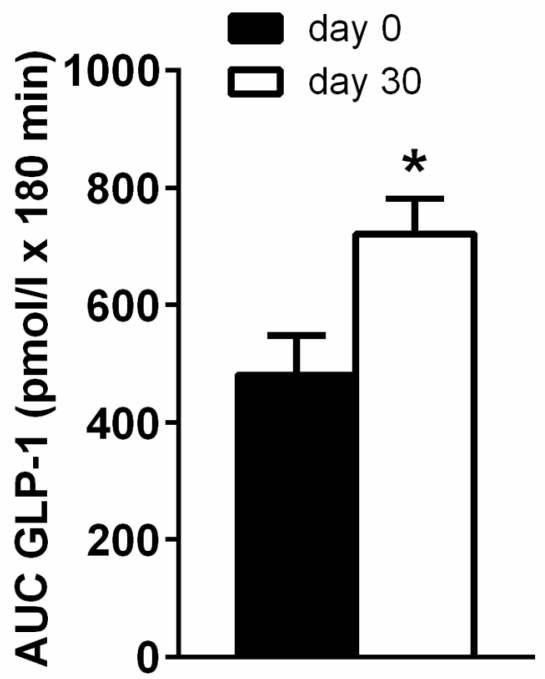

Figure 1.

(A) Plasma concentrations of active GLP-1 at day zero and after 30 days of consumption of enriched $\beta$-glucans pasta. (B) The AUC of active GLP-1 during a 3-hours monitoring period. Values are mean \pm S.E.M. $* \mathrm{p}<0.05$. ( $\mathrm{n}=10$ /group).

Ghrelin (Figure 2). Plasma ghrelin concentrations were higher in fasting state and they were reduced following the test-meals. The consumption of the $\beta$-glucans enriched pasta for 30 days significantly increased the levels of ghrelin at 0,15 and 30 minutes after intake. As shown by AUC, on the whole after 30 days treatment we observed an increased release of ghrelin. 

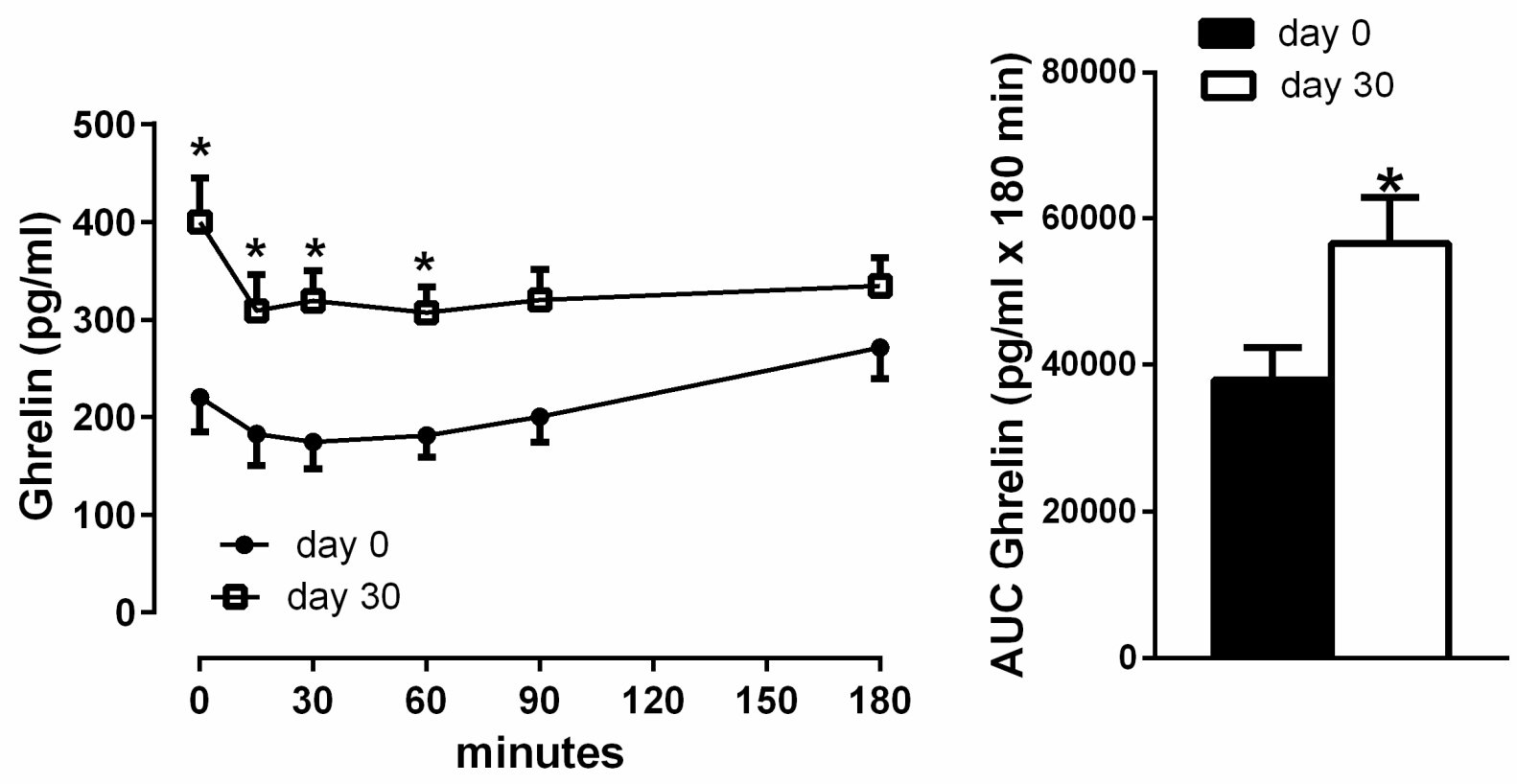

Figure 2.

(A) Plasma concentrations of ghrelin at day zero and after 30 days of consumption of enriched $\beta$-glucans pasta. (B). The AUC of ghrelin during a 3-hours monitoring period. Data are mean values \pm S.E.M. ${ }^{*} \mathrm{p}<00.05$. ( $\mathrm{n}=10$ /group).

PYY (Figure 3). The consumption of test meal induced an increase in plasma PYY concentrations starting from $15 \mathrm{~min}$ up to $90 \mathrm{~min}$, peaking at $90 \mathrm{~min}$. The consumption of the $\beta$ glucans enriched pasta for 30 days significantly increased the levels of PYY at 0, 15, 30, 60 and 90 minutes after intake. As shown by AUC, on the whole after 30 days treatment we observed an increased release of PYY. 
A

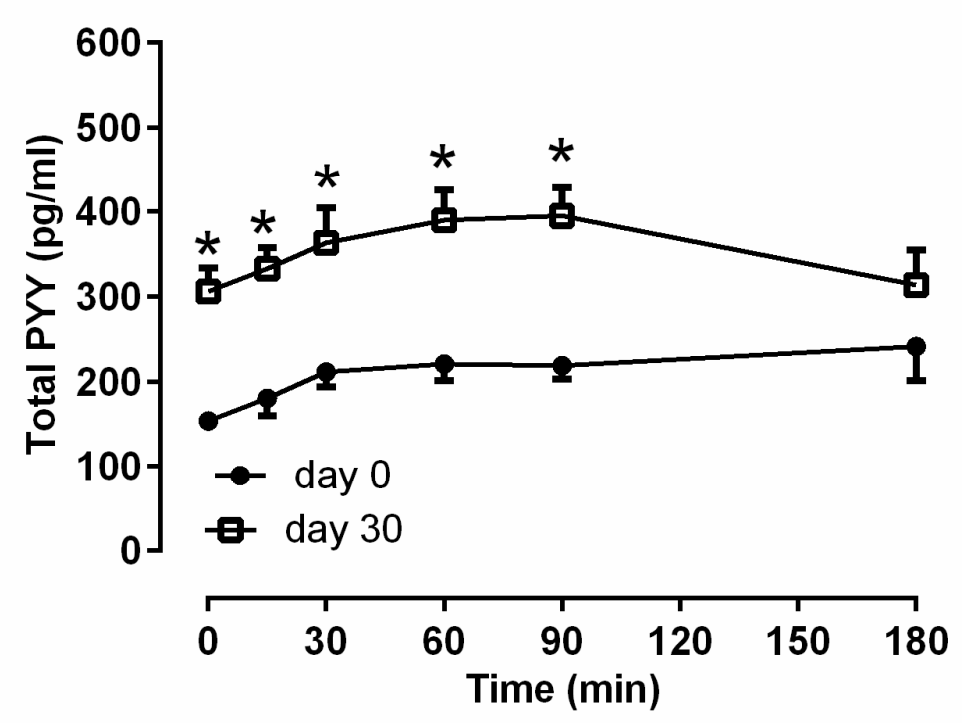

B

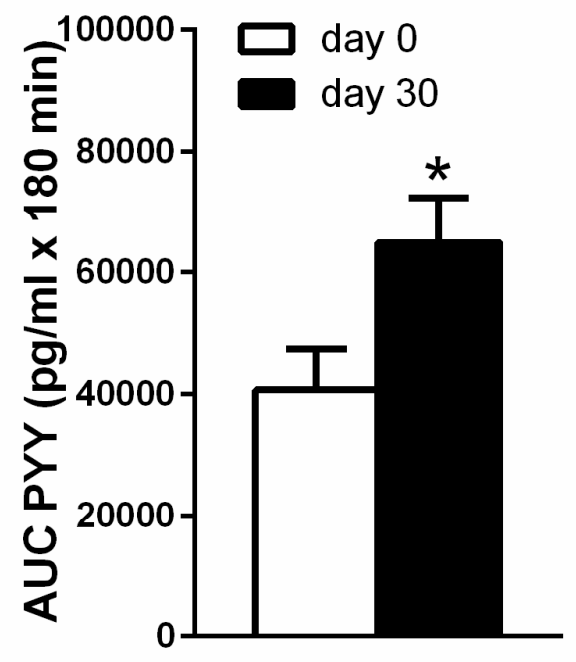

Figure 3.

(A) Plasma concentrations of PYY at day zero and after 30 days of consumption of enriched $\beta$-glucans pasta. (B) The AUC of PYY during a 3-hours monitoring period. Data are mean values \pm S.E.M. ${ }^{*} p<0.05$. ( $\mathrm{n}=10 /$ group).

GLP-2 and CCK (Figure 4). Plasma CCK concentrations were lower in fasting state and they were increased following the test-meals whereas no difference were observed for GLP-2 plasma concentrations. However, the consumption of the $\beta$-glucans enriched pasta for 30 days did not affect plasma concentrations of GLP-2 and CCK in fasting state or after the meal test. 

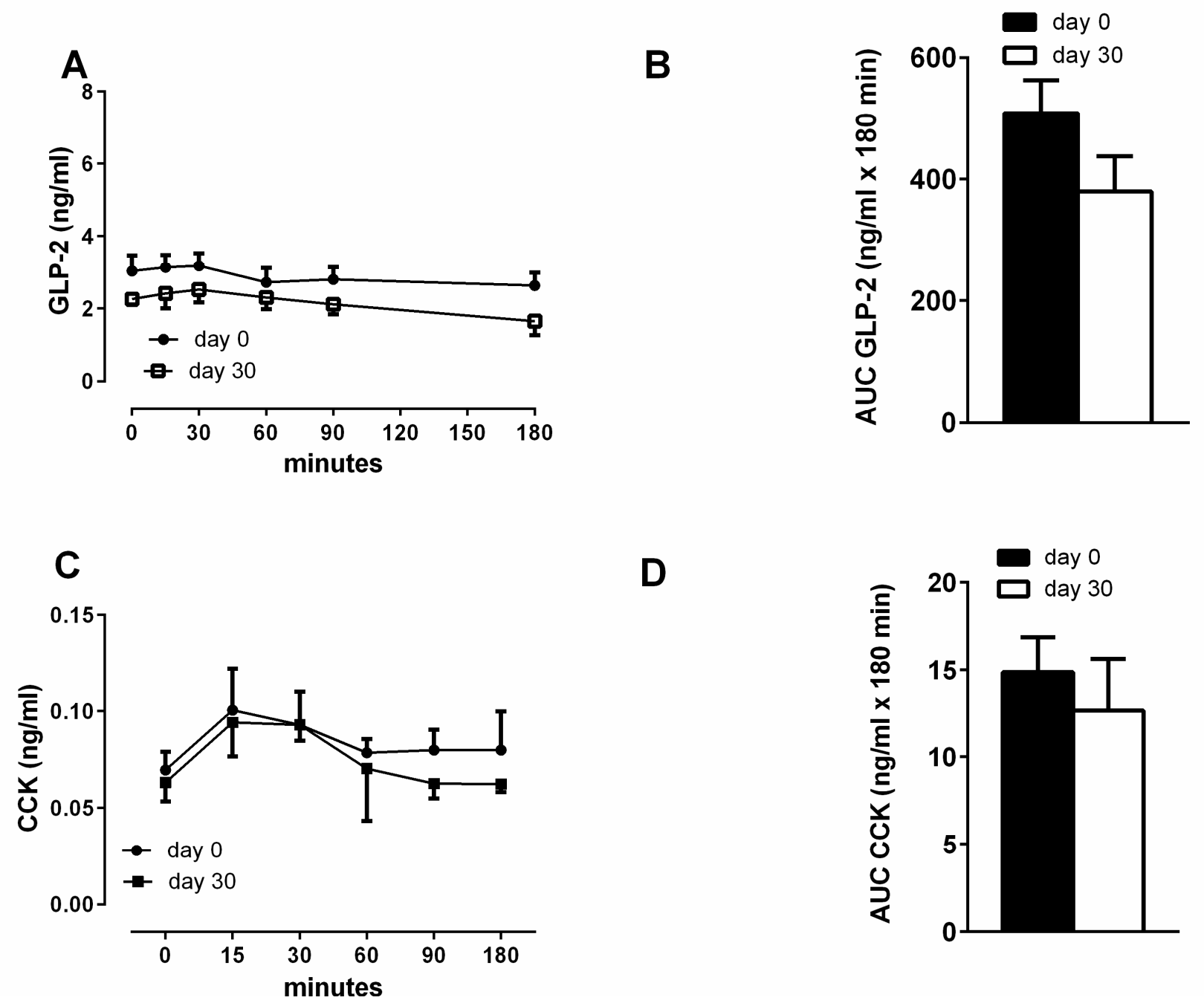

Figure 4.

(A) Plasma concentrations of GLP-2 at day zero and after 30 days of consumption of enriched $\beta$-glucans pasta. (B) The AUC of GLP-2 during a 3 -hours monitoring period. Data are mean values \pm S.E.M. (n=10/group). (C) Plasma concentrations of CKK at day zero and after 30 days of consumption of enriched $\beta$-glucans pasta. Data are mean values \pm S.E.M. (D) The AUC of CKK during a 3-hours monitoring period. Data are mean values \pm S.E.M. (n=10/group). 


\subsection{Analysis of microbiota in faeces}

The analysis of microbiota in stool samples was carried out to identify a possible variation between the baseline and the end of the nutritional intervention. No PCR inhibitors were detected in the reactions. At the end of the intervention, after 30 days, Bifidobacterium Longum, one of the most important probiotic bacterium in human intestine, increases in $80 \%$ of subjects analysed (8/10), from 2 to 36 -fold than the baseline, while did not show variation in $10 \%(1 / 10)$ and an 8 -fold decrease in the other $10 \%(1 / 10)$. Enterococcus faecum, a human pathogen responsible of many nosocomial diseases, surgical wound infection, endocarditis and urinary tract infection, showed a decrease in $90 \%$ (9/10), while no result was detected for one sample. However, these results, although interestingly, were not significant.

\section{Discussion}

The intestinal tract is the largest endocrine organ in the body secreting a wide array of hormones and neuropeptides that regulate the endocrine-metabolic answer to food ingestion within as well as outside the gut. Recent interest in gut hormones has risen with the idea that they modulate glucose tolerance and food intake through a variety of mechanisms, and such hormones are therefore excellent therapeutic candidates for the treatment of type 2 diabetes and obesity [13]. The most widely studied appetite modulating intestinal hormones are GLP-1, GLP-2, CCK, PYY and the peptide ghrelin [14-16]. So far, ghrelin is the only known circulating orexigenic satiety-related hormone and exogenous administration of ghrelin has been shown to stimulate appetite and food intake both in rats and in humans [17]. In contrast to ghrelin, GLP-1, GLP-2 and PYY are secreted into the circulation following meal ingestion and decrease with fasting [18]. The administration of GLP-1 decreases hunger and energy intake both in animals and humans [19]. The post-prandial production of GLP-1 is blunted in severely obese people but controversy exists if GLP-1 concentrations tend to normalize following body weight reduction [20,21]. Pharmacological activation of the GLP-1 system has beneficial effects on obesity-induced alterations of lipoprotein metabolism. The role of GLP-2-related pathways on lipid levels and metabolism are less clear, but some effects are opposite to GLP-1 $[22,23]$. The PYY is another intestinal hormone that is stimulated by food ingestion, especially by the fat content of meal. The infusion of PYY seems to reduce food intake in normal-weight and obese humans; in rodents, repeated infusion of PYY attenuates weight gain [16,24]. Also CCK is anorexigenic, leading to decrease in food intake [25].

In the present study, we demonstrated that pasta enriched by $6 \%$ of $\beta$-glucans strongly modified short-term hormones release by intestinal tract. In response to 30 days of pasta intake we observed different postprandial responses of ghrelin, CCK, PYY, and GLP-1. In particular, 30 days 
of regular intake of enriched $\beta$-glucans pasta significantly increased the endogenous levels of GLP-1 compared with day zero as well as those of plasma PYY levels. Furthermore, plasma ghrelin concentrations were higher in fasting state and they were reduced following the test-meals. So, food stimulated postprandial secretion of the anorexigenic hormones PYY, and GLP-1 and repression of concentrations the orexigenic hormone ghrelin that have been proposed to increase satiety and decrease short-term food intake [26]. As stated in the Introduction, it has been claimed an effect on satiety by products prepared from barley flour enriched with $\beta$-glucans, but in that study no hormonal measurement was performed. Thus, to the best of our knowledge, the present study is the first one to demonstrate an effect of intestinal hormones.

Due to the known effects of these hormones in weight regulation, we can suggest that the regular intake of pasta added with $\beta$-glucans should be a promising tool to prevent obesity.

On the other hand, it is well known that harmful metabolites, produced by the human microbiota, likely participate to ageing process, contributing to inflammageing and immunosenescence. Although the intestinal microbiota is relatively stable throughout adulthood, agerelated modifications in the intestinal tract, as well as changes in diet and host immunity reactivity, inevitably affect bacteria population composition. The prolonged retention time leads to an increase in bacterial protein fermentation and consequently putrefactive processes in the gut with a greater susceptibility to inflammatory diseases [27]. Hence, the gut ecosystem shows the potential to become a promising target for strategies able to contribute to the health status of elderly. In this context, the consumption of prebiotics as $\beta$-glucans fibres might be useful in both prevention and treatment of age-related pathophysiological conditions, favouring the attainment of longevity [13]. Accordingly, we observed changes in microbiota composition in our subjects, after 30 days treatment with pasta added with $\beta$-glucans, however, the brief period of time of our study did not allow us to reach significant changes.

It is to note that our study presents some limitations. One is strictly related to the intrinsic complexity of human as study model and to the inter/intra-individual variability. More important, we can consider this study only a pilot one due to the small size sample.

However, present and previous results clearly suggest that the regular intake of this food might useful for the prevention of obesity, type 2 diabetes and metabolic syndrome [6]. 


\section{Material and Methods}

\subsection{Study Design}

This was a $30 \pm 3$ days longitudinal, intervention study on the effect of consuming $100 \mathrm{~g}$ of pasta supplemented with $6 \%$ of $\beta$-glucans in 10 overweight young people. We took blood for hematochemical test at baseline (day zero) and after 30 days (day 30). In addition, we evaluated intestinal hormones after a challenge with test meals.

The study protocol was conducted in accordance with the Declaration of Helsinki and its amendments, and was approved by the local review board (University Hospital Ethic Committee, $\mathrm{n}$. 3024). Suitable subjects were identified from computerized clinic registers and were contacted by the clinicians personally or by telephone. All eligible candidates had to provide signed informed consent before enrolment in the study.

\subsection{Test Meal}

The intestinal hormones evaluation consisted of $100 \mathrm{~g}$ of pasta with $\beta$-glucans administered to the selected people after night fasting of day zero as sort of breakfast. Immediately after test meal blood samples were collected after 15, 30, 60, 90 and $180 \mathrm{~min}$. Intestinal hormones measured were the following GLP-1, ghrelin, PYY, CCK, and GLP-2 (Figure 5). 


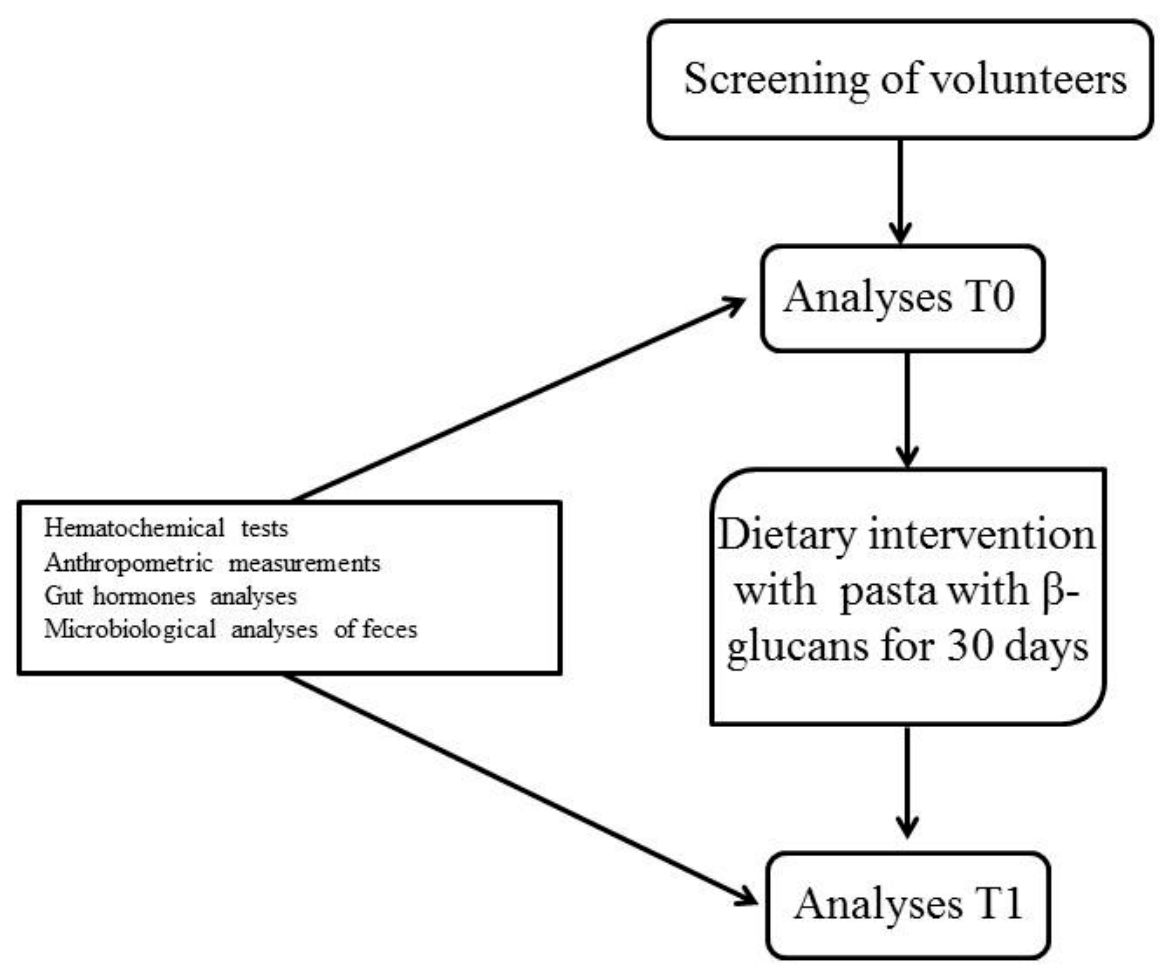

day zero=baseline day $30=$ the end of nutrition intervention

Figure 5. Flow chart of study design.

\subsection{Patients}

Inclusion criteria were: age range 20-30 years, overweight (BMI, 25-29.9 kg/m²;), normal glucose tolerance (fasting plasma glucose $<100 \mathrm{mg} / \mathrm{dL}$; glycated haemoglobin $<5.7 \%$ ), slight dyslipidemia (total cholesterol 200-240 mg/dL, LDL 130-160 mg/dL, triglyceride level $\leq 170 \mathrm{mg} / \mathrm{dL}$ ). Exclusion criteria were: any diagnosis of systemic disorder (including heart disease, hypertension, rheumatologic disease, liver, kidney and enterological disorders), any drug treatment, eating behaviour disease based on the VAS Questionnaire [28], any hypocaloric dietary treatment in the last six months. Enrolled people underwent a complete medical evaluation by a trained physicians (SB). All the subjects ended the pilot study (100\% of trial completion), none abandoned the study before completion.

Participants were asked to maintain their usual diet and physical activity level throughout the study period. Body weight, height and blood pressure of all participants were measured and blood samples and faeces were collected at day zero and at day 30. Baseline and after 30 days tests were performed between 8:00 and 9:00 a.m., in post-absorptive fasting from at least 10 hours, without any 
strenuous physical activity in the last three days. An indwelling venous cannula was placed in the forearm $10 \mathrm{~min}$ before the first blood sample. Blood samples were collected before and 15, 30, 60, 90, 180 min after the ingestion of the test meal and immediately centrifuged to collect serum samples, and stored at $-80^{\circ} \mathrm{C}$. Furthermore, faeces were stored at $-80^{\circ} \mathrm{C}$ for subsequent determinations .

\subsection{Nutritional Intervention}

$\beta$-glucans extraction, characterization and pasta have been prepared according to Montalbano et al. [29] by pasta factory Tomasello (Casteldaccia, Italy).

\subsection{Haematochemical Tests}

Plasma and serum samples were used for hematochemical test, total cholesterol, LDLcholesterol, HDL-cholesterol, alanine aminotransferase, aspartate aminotransferase, glucose, triglycerides, creatinine, gamma-glutamyl transferase, total protein, uric acid, high sensitivity creactive protein.

\subsection{Intestinal Hormones Tests}

For intestinal hormones measurement, plasma was obtained from peripheral blood sample by centrifugation at $5^{\circ} \mathrm{C}$ for $10 \mathrm{~min}$ at $3000 \mathrm{rpm}$; plasma samples were collected in prechilled EDTAcontaining tubes with apoprotein $(0.6 \mathrm{TIU} / \mathrm{ml}$ blood $)$ and dipeptidyl peptidase IV inhibitor $(10 \mu 1 / \mathrm{ml}$ blood). All samples were immediately stored at $-80^{\circ} \mathrm{C}$ until analyzed. Results were obtained from all the study participants (see flow chart Figure 5). Samples were analyzed using the following enzyme immunoassay kit: EGLP-35K for active GLP-1, EZGRT-89K for total ghrelin, EZHPPYYT66K for total PYY, EZGLP-237K for GLP-2, all from Millipore (EMD Millipore, MERCK KGaA, Darmstadt, Germany); CCK (26-33) for CCK from Phoenix (PHOENIX PHARMACEUTICALS, INC.).

\subsection{Microbiota Analysis}

In order to perform the quantification of microbiota in faeces samples at the baseline and after 30 days of nutritional intervention, the total DNA amount (bacterial and human) was extracted with the QIAamp DNA Stool Minikit (Qiagen), from a frozen stool sample of $200 \mathrm{mg}$, quantified by a spectrophotometric assay. The PCR Real_Time was performed to identify the possible variation in the amount of the following bacterial strains: Lactobacillus Fermentum, Lactobacillus acidophilus, Lactobacillus salivarius, Bifidobacterium longum, Intestinalcoccus faecium, Clostridium difficile. The amplification reactions were carried out starting from $1 \mu \mathrm{l} \mathrm{DNA}, 25 \mu \mathrm{g} / \mu \mathrm{l}$. Each assay contained 
a negative (without DNA) and a positive control (quantified DNA, specific for each strain). Moreover, specific primers and fluorescent probe labeled with the reporter dye 6-carboxyfluorescein (FAM) were used. The variation of DNA amount before and after the nutritional intervention was calculated considering the cycle threshold (CT) of each sample.

\subsection{Statistical Analysis}

Results are shown as mean \pm S.E.M. for intestinal hormones data, S.D. used for haematochemical tests. The comparison of hematochemical tests before and after the 30 days of pasta intake was performed by Wilcoxon test. The comparison between the levels of gut hormones after test meal at baseline and after 30 days was performed by ANOVA followed by Bonferroni's post-test test using Prism Version 6.0 Software (Graph Pad Software, Inc., San Diego, CA, USA). The AUC values were calculated using the trapezoidal rule. In the field of pharmacokinetics, the AUC is the area under the curve in a plot of concentration of drug in blood plasma against time. In this case, it determines whether the same standardized two formulations of test meal, before and after 30 days treatment within pasta added with $\beta$-glucans, release the same dose of hormones. A p value $<0.05$ was considered to be statistically significant.

\section{Conclusions}

The use of pasta added with $6 \% \beta$-glucans seems exhibit promising responses on glucose metabolism, LDL-cholesterol values, intestinal hormones responses and microbiota modification. 
Acknowledgments: This work was supported by PON DIMESA (Programma Operativo Nazionale Ricerca e Competitività 2007/2013 - Progetto “DI.ME.Sa.” PON02_00451_3361785. Valorisation of typical products of the Mediterranean diet and their nutraceutical use to improve health) to $\mathrm{CC}$ and $\mathrm{SV}$. AA is student of the $\mathrm{PhD}$ course directed by $\mathrm{CC}$.

Author Contributions: Design and conduction of the study :Sara Baldassano, Silvio Buscemi, Sonya Vasto; data collection and data interpretation: manuscript drafting: Sara Baldassano, Sonya Vasto. Calogero Caruso made critical revisions to the draft . All authors read and approved the final manuscript..

Conflicts of Interest: The authors have no relevant affiliations or financial involvement with any organization or entity with financial interest in or financial conflict with the subject discussed in the manuscript. 


\section{References}

1. Rieder, A.; Samuelsen A.B. Do cereal mixed-linked $\beta$-glucans possess immune-modulating activities? Mol Nutr Food Res. 2012, 56, 536-547.

2. Lazaridou, A.; Biliaderis C.G. Molecular aspects of cereal $\beta$-glucan functionality: physical properties, technological applications and physiologycal effects. J Cereal Sci. 2007, 46, 101-118.

3. Gibbons, C.; Caudwell, P.; Finlayson, G.; Webb, D.L.; Hellström, P.M.; Näslund, E.; Blundell, J.E. Comparison of postprandial profiles of ghrelin, active GLP-1, and total PYY to meals varying in fat and carbohydrate and their association with hunger and the phases of satiety. $J$ Clin Endocrinol Metab. 2013, 98, E847-855.

4. Zijlstra, N., Mars, M.; de Wijk, R.A.; Westerterp-Plantenga, M.S.; de Graaf, C. The effect of viscosity on ad libitum food intake. Int J Obes. 2008, 32, 676-683.

5. Barone Lumaga, R.; Azzali, D.; Fogliano, V.; Scalfi, L.; Vitaglione, P. Sugar and dietary fibre composition influence, by different hormonal response, the satiating capacity of a fruit-based and a $\beta$-glucan-enriched beverage. Food Funct. 2012, 3, 67-75.

6. Barera, A.; Buscemi, S.; Monastero, R.; Caruso, C.; Caldarella, R.; Ciaccio, M.; Vasto, S. $\beta$ glucans: ex vivo inflammatory and oxidative stress results after pasta intake. Immun Ageing. 2016, 7, 13:14.

7. Aiello, A.; Accardi, G., Candore, G.; Carruba, G.; Davinelli, S.; Passarino, G.; Scapagnini G.; Vasto, S.; Caruso C. Nutrigerontology: a key for achieving successful ageing and longevity. Immun Ageing. 2016, 21, 13:17.

8. Pentikäinen, S.; Karhunen, L.; Flander, L.; Katina, K.; Meynier, A.; Aymard, P.; Vinoy, S.; Poutanen, K. Enrichment of biscuits and juice with oat $\beta$-glucan enhances postprandial satiety. Appetite. 2014, 75, 150-156.

9. Ley, R.E.; Turnbaugh, P.J.; Klein, S.; Gordon, J.I. Microbial ecology: human gut microbes associated with obesity. Nature. 2006, 7122, 1022-1023.

10. Cani, P.D.; Amar, J.; Iglesias, M.A.; Poggi, M.; Knauf, C.; Bastelica, D.; Neyrinck, A.M.; Fava, F.; Tuohy, K.M.; Chabo, C.; Waget, A.; Delmée, E.; Cousin, B.; Sulpice, T.; Chamontin, B.; Ferrières, J.; Tanti, J.F.; Gibson, G.R.; Casteilla, L.; Delzenne, N.M.; Alessi, M.C.; Burcelin, R. Metabolic endotoxemia initiates obesity and insulin resistance. Diabetes. 2007, 56, 1761-72.

11. Cani, P.D.; Neyrinck, A.M.; Fava, F.; Knauf, C.; Burcelin, R.G.; Tuohy, K.M.; Gibson, G.R.; Delzenne, N.M. Selective increases of bifidobacteria in gut microflora improve high-fat-dietinduced diabetes in mice through a mechanism associated with endotoxaemia. Diabetologia. 2007, 50, 2374-2383.

12. Wichmann, A.; Allahyar, A.; Greiner, T.U.; Plovier, H.; Lundén, G.Ö.; Larsson, T.; Drucker, D.J.; Delzenne, N.M.; Cani, P.D.; Bäckhed, F. Microbial modulation of energy availability in the colon regulates intestinal transit. Cell Host Microbe. 2013, 14, 582-590.

13. Wren, A.M.; Bloom, R. Gut hormones and appetite control. Gastroenterology. 2007, 132, 21162130. 
14. Lean, M.E.; Malkova, D. Altered gut and adipose tissue hormones in overweight and obese individuals: cause or consequence? Int J Obes (Lond). 2016, 40, 622-623.

15. Horner, K.; Lee, S. Appetite-related peptides in childhood and adolescence: role of ghrelin, PYY, and GLP-1. Appl Physiol Nutr Metab. 2015, 40, 1089-1099.

16. Batterham, R.L.; Cowley, M.A.; Small, C.J.; Herzog, H.; Cohen, M.A.; Dakin, C.L.; Wren, A.M.; Brynes, A.E.; Low, M.J.; Ghatei, M.A.; Cone, R.D.; Bloom, S.R. Gut hormone PYY(3-36) physiologically inhibits food intake. Nature. 2002, 418, 650-654.

17. Druce, M.R.; Wren, A.M.; Park, A.J.; Milton, J.E.; Patterson, M.; Frost, G.; Ghatei, M.A. Small C.; Bloom, S.R. Ghrelin increases food intake in obese as well as lean subjects. Int J Obes (Lond). 2005, 29, 1130-1136.

18. Cummings, D.; Frayo, R.S.; Marmonier, C.; Aubert, R.; Chapelot, D. Plasma ghrelin levels and hunger scores in humans initiating meals voluntarily without time-and food-related cues. Am $J$ Physiol Endocrinol Metab. 2004, 287, E297-E304.

19. Näslund, E.; Barkeling, B.; King, N.; Gutniak, M.; Blundell, J.E.; Holst, J.J.; Rössner, S.; Hellström, P.M. Energy intake and appetite are suppressed by glucagon-like peptide-1 (GLP-1) in obese men. Int J Obes Relat Metab Disord. 1999, 23, 304-311.

20. Drucker, D.J.; Nauck, M.A. The incretin system: glucagon-like peptide-1 agonists and dipeptidyl peptidase-4 inhibitors in type 2 diabetes. Lancet. 2006, 368, 1696-1705.

21. Gibbons, C.; Caudwell, P.; Finlayson, G.; Webb, D.L.; Hellström, P.M.; Näslund, E.; Blundell, J.E. Comparison of postprandial profiles of ghrelin, active GLP-1, and total PYY to meals varying in fat and carbohydrate and their association with hunger and the phases of satiety. $J$ Clin Endocrinol Metab. 2013, 98, E847-855.

22. Lutz, T.A.; Osto, E. Glucagon-like peptide-1, glucagon-like peptide-2, and lipid metabolism. Curr Opin Lipidol. 2016, 27, 257-263.

23. Feinle, C.; O'Donovan, D.; Doran, S.; Andrews, J.M.; Wishart, J.; Chapman, I.; Horowitz, M. Effects of fat digestion on appetite, APD motility, and gut hormones in response to duodenal fat infusion in humans. Am J Physiol Gastrointest Liver Physiol. 2003, 284, G798-807.

24. Zwirska-Korczala, K.; Konturek, S.J.; Sodowski, M.; Wylezol, M.; Kuka, D.; Sowa P.; Adamczyk-Sowa, M,; Kukla, M.; Berdowska, A.; Rehfeld J.F.; Bielanski, W.; Brzozowski, T. Basal and postprandial plasma levels of PYY, ghrelin, cholecystokinin, gastrin and insulin in women with moderate and morbid obesity and metabolic syndrome. J Physiol Pharmacol. 2007, Suppl 1, 13-35.

25. Marić, G.; Gazibara, T.; Zaletel, I.; Labudović Borović, M.; Tomanović, N.; Ćirić, M.; Puškaš, N. The role of gut hormones in appetite regulation. Acta Physiol Hung. 2014, 101, 395-407.

26. Chungchunlam, S.M.; Henare, S.J.; Ganesh, S.; Moughan, P.J. Dietary whey protein influences plasma satiety-related hormones and plasma amino acids in normal-weight adult women. Eur $J$ Clin Nutr. 2015, 69, 179-186. 
27. Woodmansey, E. Intestinal bacteria and ageing. J Appl Microbiol. 2007, 102, 1178-1186.

28. Rogers, P.J.; Blundell, J.E. Effect of anorexic drugs on food intake and the micro-structure of eating in human subjects. Psychopharmacology (Berl). 1979, 66, 159-165.

29. Montalbano, A.; Tesoriere, L.; Diana, P.; Barraja, P.; Carbone, A.; Spanò, V.; Parrino, B.; Attanzio, A.; Livrea, M.A.; Cascioferro S.; Cirrincione, G. Quality characteristics and in vitro digestibility study of barley flour enriched ditalini pasta. LWT-Food Science and Technology. 2016, 72, 223-228.

(C) 2016 by the authors; licensee Preprints, Basel, Switzerland. This article is an open access article distributed under the terms and conditions of the Creative Commons by Attribution (CC-BY)

license (http://creativecommons.org/licenses/by/4.0/). 